\title{
Production asymmetry of open charm mesons within unfavoured fragmentation scenario
}

\author{
Rafał Maciuła ${ }^{1, *}$ \\ ${ }^{1}$ Institute of Nuclear Physics, Polish Academy of Sciences, Radzikowskiego 152, PL-31-342 Kraków, \\ Poland
}

\begin{abstract}
We consider unfavoured light quark/antiquark to $D$ meson fragmentation. We discuss nonperturbative effects for small transverse momenta. The asymmetry for $D^{+}$and $D^{-}$production measured by the $\mathrm{LHCb}$ collaboration provides natural constraints on the parton (quark/antiquark) fragmentation functions. We find that already a fraction of $q / \bar{q} \rightarrow D$ fragmentation probability is sufficient to account for the measured asymmetry. Large $D$-meson production asymmetries are found for large $x_{F}$ which is related to dominance of light quark/antiquark $q / \bar{q} \rightarrow D$ fragmentation over the standard $c \rightarrow D$ fragmentation. As a consequence, prompt atmospheric neutrino flux at high neutrino energies can be much larger than for the conventional $c \rightarrow D$ fragmentation. The latter can constitute a sizeable background for the cosmic neutrinos claimed to be observed recently by the IceCube Observatory.
\end{abstract}

\section{Introduction}

It is believed that the $D$ mesons are produced dominantly via $c \rightarrow D$ fragmentation mechanism which leads to a symmetric production of mesons and anti-mesons. However, asymmetries for $D^{+}$and $D^{-}$production were obtained recently at the LHC for $p-p$ collisions [1]. The LHCb collaboration found rather small asymmetries of the order of $1 \%$, but, the mechanism behind the effect is still not under theoretical control.

The production asymmetries were interpreted in Ref. [2] as due to meson cloud mechanism and specific structure of the proton Fock components. The string model approach to the problem of heavy meson production and asymmetries in the production of heavy mesons was discussed in extent in Ref. [3]. The LHCb asymmetry was discussed also in the framework of heavy-quark recombination approach [4]. Here there are four unknown parameters responsible for formation of $D$ mesons. It was shown that with some combination of parameters one can describe the LHCb data [4].

The conventional $D$ meson production mechanism leads to symmetry in $D^{+} / D^{-}$or $D^{0} / \bar{D}^{0}$ production, i.e. $\sigma\left(D^{+}\right)=\sigma\left(D^{-}\right)$. Here we consider a simple alternative phenomenological explanation using so-called unfavored fragmentation functions responsible for light quark/antiquark fragmentation to $D$ mesons. Such unfavoured fragmentation functions are known to be important e.g. for $K^{+} / K^{-}$production and corresponding asymmetries obtained at SPS [5] and RHIC [6]. Such asymmetries for kaon production were nicely explained in the picture of subleading parton fragmentation at low energies [7]. It is interesting whether the

\footnotetext{
*e-mail: rafal.maciula@ifj.edu.pl
} 
unfavoured fragmentation functions can describe the observed experimentally asymmetries in proton-proton collisions.

In the present paper we wish to constrain the strength of $q \rightarrow D(\bar{q} \rightarrow D)$ fragmentation functions using the recent $\mathrm{LHCb}$ data for $D^{+} / D^{-}$asymmetry. Then we shall discuss $q / \bar{q} \rightarrow$ $D^{ \pm}$contribution to $d \sigma / d x_{F}$ distributions. Possible consequences for prompt atmospheric neutrino production will be discussed.

\section{A theoretical basis of the calculations}

We calculate the dominant at large $x_{F}$ high-energy processes: $u g \rightarrow u g, d g \rightarrow d g, \bar{u} g \rightarrow \bar{u} g$ and $\bar{d} g \rightarrow \bar{d} g$ and subsequent light quark/antiquark to D meson fragmentation and/or decays. The calculations are done in the leading-order (LO) collinear factorization approach with a special treatment of minijets at low transverse momenta, as adopted in РүтніA, by multiplying standard cross section by a somewhat arbitrary suppression factor $F_{s u p}\left(p_{T}\right)=\frac{p_{T}^{4}}{\left(\left(p_{T}^{0}\right)^{2}+p_{T}^{2}\right)^{2}}[8]$. In this calculation we use the MMHT2014lo PDFs. The factorization and renormalization scales are taken as: $\mu_{F}^{2}, \mu_{R}^{2}=\mu_{0}^{2}+p_{T}^{2}$ with $\mu_{0}^{2}=0.5^{2} \mathrm{GeV}^{2}$.

After parton-level calculations, we have to include $u, \bar{u}, d, \bar{d} \rightarrow D^{i}$ parton fragmentation. The corresponding fragmentation functions fulfill the following flavour symmetry conditions:

$$
D_{d \rightarrow D^{-}}(z)=D_{\bar{d} \rightarrow D^{+}}(z)=D^{(0)}(z) .
$$

Similar symmetry relations hold for fragmentation of $u$ and $\bar{u}$ to $D^{0}$ and $\bar{D}^{0}$ mesons. However $D_{q \rightarrow D^{0}}(z) \neq D_{q \rightarrow D^{+}}(z)$ which is caused by the contributions from decays of vector $D^{*}$ mesons. Furthermore we assume for doubly suppressed fragmentations:

$$
D_{\bar{u} \rightarrow D^{ \pm}}(z)=D_{u \rightarrow D^{ \pm}}(z)=0 .
$$

We can parametrize the unfavoured fragmentation functions in this phase space region as:

$$
D_{q \rightarrow D}(z)=A_{\alpha}(1-z)^{\alpha} .
$$

Instead of fixing the uknown $A_{\alpha}$ we will operate rather with the fragmentation probability:

$$
P_{q \rightarrow D}=\int d z A_{\alpha}(1-z)^{\alpha}
$$

and calculate corresponding $A_{\alpha}$ for a fixed $P_{q \rightarrow D}$ and $\alpha$. Therefore in our effective approach we have only two free parameters.

Another simple option one could consider is:

$$
D_{q_{f} \rightarrow D}(z)=P_{q_{f} \rightarrow D} \cdot D_{\text {Peterson }}(1-z) .
$$

Then $P_{q_{f} \rightarrow D}$ would be the only free parameter. For heavy quark fragmentation $(c \rightarrow D)$ the Peterson fragmentation function is peaked at large $z$. However, the light quark/antiquark fragmentation is expected to be dominant at small $z$. This is the case of Peterson fragmentation function reflected with respect to $z=1 / 2$. We use such a function purely phenomenologically to test uncertainties related to the shape of the a priori unknown function.

In addition to the direct fragmentation (given by $D^{(0)}(z)$ ) there are also contributions with intermediate vector $D^{*}$ mesons. Including both direct and resonant contributions the combined fragmentation function of light quarks to charged $D$ mesons can be written as:

$$
D_{d / \bar{d} \rightarrow D^{\mp}}^{\mathrm{eff}}(z)=D_{d / \bar{d} \rightarrow D^{\mp}}^{0}(z)+P_{\mp \rightarrow \mp} \cdot D_{d / \bar{d} \rightarrow D^{*, \mp}}^{1}(z) .
$$


The decay branching ratios can be found in Ref. [9] and is $P_{ \pm \rightarrow \pm}=0.323$. The indirect vector meson contributions have the same flavour structure as the direct one. It is easy to check that the decay $D^{*} \rightarrow D X$ practically does not change the distribution in $z$.

We assume flavour symmetry of fragmentation functions also for vector $D$ meson:

$$
D_{u / \bar{u} \rightarrow \bar{D}^{*, 0} / D^{*, 0}}^{1}(z)=D_{d / \bar{d} \rightarrow D^{*,}}^{1}(z)=D^{(1)}(z) .
$$

Finally we shall take an approximation:

$$
D^{(0)}(z) \approx D^{(1)}(z)
$$

which can be easily relaxed if needed. We think that such an approximation is, however, sufficient for the present exploratory calculations.

\section{The LHCb production asymmetry}

The flavour asymmetry in production is defined as $A_{D^{+} / D^{-}}(\xi)=\frac{\frac{d \sigma_{D^{-}}}{d \xi}(\xi)-\frac{d \sigma_{D^{+}}}{d \xi}(\xi)}{\frac{d \sigma_{D^{-}}}{d \xi}(\xi)+\frac{d \sigma_{D^{-}}}{d \xi}(\xi)}$, where $\xi=\eta, p_{T}$. To calculate asymmetry we have to include also dominant contribution corresponding to conventional $c / \bar{c} \rightarrow D / \bar{D}$ fragmentation. The leading-order pQCD calculation is not reliable in this context. In the following the conventional contribution is calculated within the $k_{t}$-factorization approach with the Kimber-Martin-Ryskin unintegrated parton distributions which has proven to well describe the LHC data [10]. Such an approach seems consistent with collinear next-to-leading order approach (see e.g. a discussion in Ref. [11]).

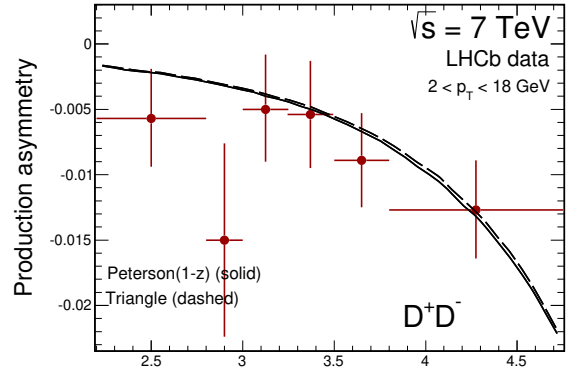

$\eta$

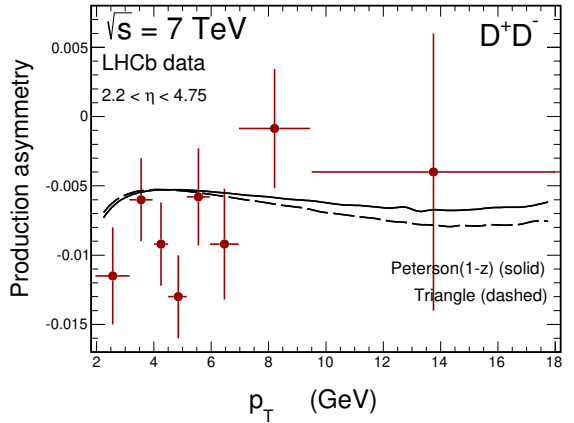

$\mathrm{p}_{\mathrm{T}}(\mathrm{GeV})$

Figure 1. $A_{D^{+} / D^{-}}$production asymmetry measured by the LHCb collaboration at $\sqrt{s}=7 \mathrm{TeV}$ as a function of $D$ meson pseudorapidity (left panel) and $D$ meson transverse momentum (right panel).

In Fig. 1 we show results for the asymmetry for $P_{q \rightarrow D}$ adjusted to the LHCb data. In this calculation, and in the rest of the paper, we have fixed $\alpha=1$ in formula (3). We shall call corresponding fragmentation functions as triangular for brevity. In the left panel we show $A_{D^{+} / D^{-}}(\eta)$ for $p_{T, D} \in(2,18) \mathrm{GeV}$ and in the right panel we show $A_{D^{+} / D^{-}}\left(p_{T}\right)$ for $2.2<\eta<$ 4.75. We find that $P_{q \rightarrow D}=0.005 \pm 0.001$ for triangle fragmentation function and $P_{q \rightarrow D}=$ $0.007 \pm 0.001$ for Peterson $(1-\mathrm{z})$ is consistent with main trends of the LHCb data. This are rather small numbers compared to $c / \bar{c} \rightarrow D / \bar{D}$ fragmentation which happens with probability of the order of $50 \%$. The results do not depend on transverse momentum cut $p_{T}^{0}$, since the $\mathrm{LHCb}$ kinematics excludes the uncertain region of very small meson transverse momenta. 


\section{Cosequences for prompt atmospheric neutrinos}

In this subsection we wish to show results relevant for high-energy prompt atmospheric neutrinos. As discussed recently in Ref. [12] a rather large $x_{F} \sim 0.5$ region is important in this context. The $d \sigma / d x_{F}$ distribution of mesons is the most appropriate distribution in this context.
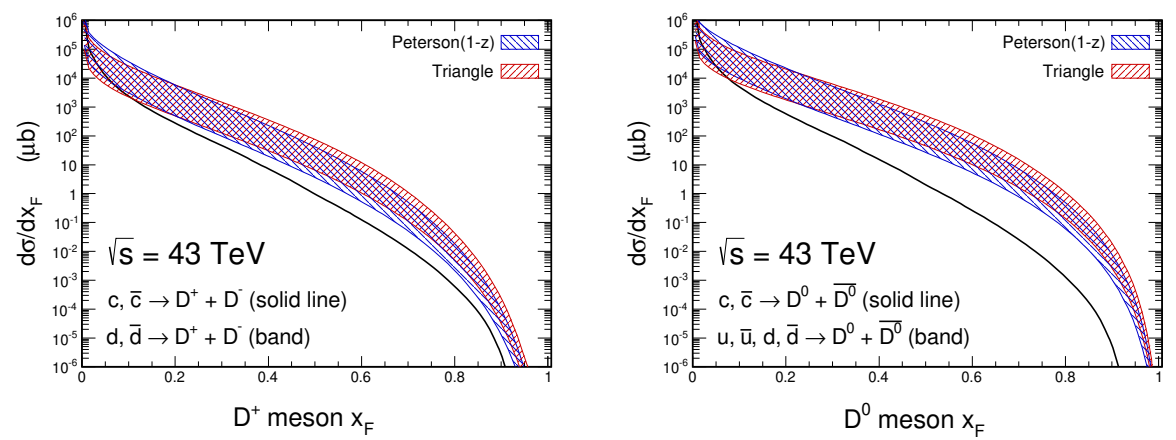

Figure 2. Distribution in $x_{F}$ for charged $D^{+}+D^{-}$(left panel) and neutral $D^{0}+\bar{D}^{0}$ (right panel) $D$ mesons from conventional (solid lines) and subleading (shaded bands) mechanisms.

In Fig. 2 we compare the two contributions: (a) conventional one corresponding to $c \rightarrow D$ fragmentation and (b) subleading one corresponding to $q \rightarrow D$ fragmentation, for the sum of $D^{+}+D^{-}$(left panel) and $D^{0}+\overline{D^{0}}$ (right panel) mesons for $\sqrt{s}=43 \mathrm{TeV}$. While at small $x_{F}$ the conventional contribution dominates, at large $x_{F}$ the situation reverses. In addition we show the uncertainties bands where the upper and lower limits correspond to the predictions for $p_{T}^{0}=0.5$ and $1.5 \mathrm{GeV}$, respectively. The enhancement due to the subleading contributions for neutral $D$ meson seems bigger than that for charged $D$ mesons. For example, for the triangle fragmentation functions, at $\sqrt{s}=43 \mathrm{TeV}$ for $x_{F} \sim 0.5$ the cross section for charged mesons $\left(D^{+}+D^{-}\right)$is $3-15$ times bigger than for conventional approach while the cross section for neutral mesons $\left(D^{0}+\bar{D}^{0}\right)$ is $20-200$ times bigger.

This study was partially supported by the Polish National Science Center grant DEC2014/15/B/ST2/02528.

\section{References}

[1] R. Aaij et al. [LHCb Collaboration], Phys. Lett. B 718, 902 (2013)

[2] E.R. Cazaroto, et al., Phys. Lett. B724, 108 (2013)

[3] E. Norrbin and T. Sjöstrand, Eur. Phys. J. C17, 137 (2000)

[4] W.K. Lai, A.K. Leibovich and A.A. Petrov, Phys. Rev. D90, 054022 (2014)

[5] T. Anticic et al. [NA49 Collaboration], Eur. Phys. J. C 68, 1 (2010)

[6] I. Arsene et al. (BRAHMS collaboration), Phys. Rev. Lett. 98, 252001 (2007)

[7] M. Czech and A. Szczurek, Phys. Rev. C72 (2005) 015202; J. Phys. G32, 1253 (2006)

[8] T. Sjöstrand et al., Comput. Phys. Commun. 191, 159 (2015)

[9] C. Patrignani et al. [Particle Data Group], Chin. Phys. C 40, no. 10, 100001 (2016)

[10] R. Maciuła and A. Szczurek, Phys. Rev. D87, 094022 (2013)

[11] R. Maciuła and A. Szczurek, Phys. Rev. D94, 114037 (2016)

[12] V. P. Goncalves, R. Maciuła, R. Pasechnik and A. Szczurek, Phys. Rev. D 96, no. 9, 094026 (2017) 